\title{
BMJ Open Cost-effectiveness of pembrolizumab for treatment of platinum-resistant recurrent or metastatic head and neck squamous cell carcinoma in China: an economic analysis based on a randomised, open-label, phase III trial
}

\author{
Wenxiu Xin (D) , ${ }^{1,2}$ Haiying Ding, ${ }^{1,2}$ Qilu Fang, ${ }^{1,2}$ Xiaowei Zheng, ${ }^{1,2}$ \\ Yinghui Tong (1) , 1,2 Gaoqi Xu, ${ }^{1,2}$ Guonong Yang ${ }^{1,2}$
}

To cite: Xin W, Ding $H$, Fang $Q$, et al. Cost-effectiveness of pembrolizumab for treatment of platinum-resistant recurrent or metastatic head and neck squamous cell carcinoma in China: an economic analysis based on a randomised, openlabel, phase III trial. BMJ Open 2020;10:e038867. doi:10.1136/ bmjopen-2020-038867

- Prepublication history and additional material for this paper are available online. To view these files, please visit the journal online (http://dx.doi. org/10.1136/bmjopen-2020038867).

WX and HD contributed equally.

Received 27 March 2020

Revised 03 August 2020

Accepted 23 November 2020

Check for updates

(c) Author(s) (or their employer(s)) 2020. Re-use permitted under CC BY-NC. No commercial re-use. See rights and permissions. Published by BMJ.

For numbered affiliations see end of article.

Correspondence to Mrs Guonong Yang; yanggn@zjcc.org.cn and Dr Wenxiu Xin;

xinwx@zjcc.org.cn

\section{ABSTRACT}

Background Pembrolizumab was recently demonstrated to have survival benefit in patients with recurrent or metastatic head and neck squamous cell carcinoma ( $r /$ mHNSCC). However, the cost-effectiveness of pembrolizumab versus chemotherapy in China remains uncertain.

Objective This analysis aimed to describe the costeffectiveness of pembrolizumab versus standard-of-care (SOC) therapy in r/mHNSCC in China.

Design A Markov model consisting of three health states (stable, progressive and dead) was developed to compare the cost and effectiveness of pembrolizumab with SOC in platinum-resistant $r / m H N S C C$. Model inputs for transition probabilities and toxicity were collected from the KEYNOTE-040 trial, while health utilities were estimated from a literature review. Cost data were acquired for the payer's perspective in China. Costs and outcomes were discounted at an annual rate of $3.0 \%$. Sensitivity analyses were conducted to test the uncertainties surrounding model parameters.

Outcome measures The primary outcome was incremental cost-effectiveness ratios (ICERs), which were calculated as the cost per quality-adjusted life years (QALYS).

Results The total mean cost of pembrolizumab and SOC was US $\$ 45861$ and US\$41 950, respectively. As for effectiveness, pembrolizumab yielded 0.31 QALYS compared with 0.25 QALYs for SOC therapy. The ICER for pembrolizumab versus SOC was US\$65 186/QALY, which was higher than the willingness-to-pay threshold (WTP) of US\$28 130/QALY in China. The univariate sensitivity analysis indicated that utility values for progressive state, probability from stable to progressive in the SOC group, as well as cost of pembrolizumab were the three most influential variables on ICER. The probabilistic sensitivity analysis demonstrated that standard therapy was more likely to be cost-effective compared with pembrolizumab at a WTP value of US\$28 130/QALY. Results were robust across both univariate analysis and probabilistic sensitivity analysis.
Strengths and limitations of this study

- Pembrolizumab was recently approved to have survival benefit in patients with recurrent or metastatic head and neck squamous cell carcinoma $(\mathrm{r} / \mathrm{mHN}$ SCC); however, the cost-effectiveness of pembrolizumab in treating $\mathrm{r} / \mathrm{mHNSCC}$ remains unknown.

- To our knowledge, this is the first cost-effectiveness analysis comparing pembrolizumab with methotrexate, docetaxel or cetuximab in treating patients with $\mathrm{r} / \mathrm{mHNSCC}$.

- The main limitation of the study is that resource use in clinical trials may not represent resources in real clinical practice since clinical trials are conducted in a selected population meeting the inclusion and exclusion criteria.

Conclusions Pembrolizumab is not likely to be a costeffective strategy compared with SOC therapy in patients with platinum-resistant r/mHNSCC in China.

Trial registration number NCT02252042; Post-results.

\section{INTRODUCTION}

Head and neck squamous cell carcinoma (HNSCC) represents a major global cause of cancer-associated morbidity and death, with a worldwide incidence of 550000 cases and 380000 deaths per year. ${ }^{12}$ After definitive treatment, approximately 30\%-40\% of patients with HNSCC will progress ${ }^{34}$ and about $50 \%-60 \%$ will have recurrent disease. ${ }^{5}$ Platinum-based systemic chemotherapy regimens are commonly used in the first-line treatment of patients with recurrent or metastatic HNSCC ( $\mathrm{r} / \mathrm{mHNSCC})$. For patients with failure of first-line platinum therapy, the commonly used drug is methotrexate. ${ }^{6}$ The second-line drugs paclitaxel or docetaxel 
have a certain salvage effect if the first-line therapy does not receive the taxane. ${ }^{78}$ Cetuximab is also suitable for patients who have not been exposed to this drug or have poor Karnofsky performance status (KPS) score. ${ }^{9}$

Checkpoint inhibitors of the programmed cell death protein 1 (PD-1) have shown impressive effects on a number of cancers. ${ }^{10-13}$ In recent years, anti-PD-1 drugs have developed rapidly in advanced HNSCC. The Food and Drug Administration (FDA) of the USA continuously approved the indications of pembrolizumab and nivolumab for treatment of recurrent or metastatic HNSCC. Indications of pembrolizumab approved by the China Food and Drug Administration include melanoma, non-small cell lung cancer and oesophageal cancer. Besides, due to the excellent tumour treatment effect, pembrolizumab is also widely used in HNSCC, small cell lung cancer, classic Hodgkin's lymphoma, primary mediastinal large B cell lymphoma, urothelial carcinoma, gastric cancer, cervical cancer, colorectal cancer and many other cancer types according to the recommendations for indications approved by FDA and several guidelines such as the National Comprehensive Cancer Network and the Chinese Society of Clinical Oncology.

The recently reported KEYNOTE- $040^{14}$ study found a survival benefit in patients with platinum-resistant recurrent or metastatic disease who received pembrolizumab. In this clinical trial, patients were randomly assigned to receive pembrolizumab or standard-of-care (SOC) (docetaxel, methotrexate, or cetuximab). The study showed that the median overall survival was 8.4 months (95\% CI 6.4 to 9.4 ) in the pembrolizumab group and 6.9 months (95\% CI 5.9 to 8.0 ) in the SOC group. Thus, pembrolizumab extended the median overall survival by 1.5 months. Also, patients in the pembrolizumab group had a favourable safety profile compared with patients in the SOC group. Although there is a significant improvement in the treatment of patients with $\mathrm{r}$ /mHNSCC, the prognosis remains relatively poor and the economic value of pembrolizumab in this population remains unknown. The objective of this study was to evaluate the costeffectiveness of pembrolizumab compared with standard treatment in order to find a more cost-effective therapy in the treatment of $\mathrm{r} / \mathrm{mHNSCC}$ in China.

\section{METHODS}

\section{Trial background}

The target patients in the model were in line with the eligibility criteria for the randomised, open-label, phase III clinical trial (KEYNOTE-040). This included patients with HNSCC that progressed during or after platinumcontaining therapy for recurrent and/or metastatic disease. Patients that recurred or progressed within 3-6 months of platinum-containing therapy for locally advanced disease were also included. Patients were randomly assigned to receive either pembrolizumab or investigator's choice of SOC therapy. The same treatment mixes as in the SOC arm (26.2\% methotrexate, $44.4 \%$ docetaxel, 29.4\% cetuximab) were assumed in our model without adoptions, as these represent standard of care. Patients assigned to the pembrolizumab arm received 200 mg every 3 weeks intravenously. In the SOC arm, patients received $40 \mathrm{mg} / \mathrm{m}^{2}$ body surface area of methotrexate per week intravenously (could be increased to $60 \mathrm{mg} / \mathrm{m}^{2}$ in the absence of toxicity), $75 \mathrm{mg} / \mathrm{m}^{2}$ of docetaxel every 3 weeks intravenously, or $250 \mathrm{mg} / \mathrm{m}^{2}$ of cetuximab per week intravenously following a loading dose of $400 \mathrm{mg}$ / $\mathrm{m}^{2}$. Treatment continued until progression was confirmed on a scan obtained at least 4 weeks later or other criteria requiring discontinuation were met.

\section{Patient and public involvement}

There was patient representation in the KEYNOTE-040 trial. However, patients or the public were not involved in this cost-effectiveness analysis.

\section{Model structure}

We compared the cost-effectiveness of pembrolizumab with methotrexate, docetaxel or cetuximab in patients with platinum-resistant $\mathrm{r} / \mathrm{mHNSCC}$. We conducted a Markov model using TreeAge Pro Suite (TreeAge Software, Williamstown, Massachusetts, USA) to simulate treatments, adverse events, costs, survival and quality of life among simulated patients (figure 1). The abbreviated decision tree and Markov model are presented in figure 1A. Three mutually exclusive health states, progression-free (stable state), progressive disease (cancer progression) and death, were included in the state transition diagram (figure 1B). Patients started receiving pembrolizumab or standard chemotherapy in the stable state and could stay in or move to progressive disease or death at a cycle length due to their assigned transition probabilities. The simulation was conducted in 3-week cycles for a period of 30 years, during which all patients were expected to die. Transition probabilities of every state were calculated based on the following equation: $\mathrm{P}(1$ month $)=1-00.5^{\text {(1/median time to event })}$. The equation was derived from $\mathrm{P}=1-\mathrm{e}^{-\mathrm{R}}$ and $\mathrm{R}=-\ln (0.5) /($ time to event/ number of treatment cycles). ${ }^{15-17}$ The modelled overall survival curve is presented in online supplemental figure 1A. The survival curve extracted using Engauge Digitizer software (V.4.1; http://digitizer.sourceforge.net) from clinical trials is shown in online supplemental figure 1B.

\section{Cost}

Since the therapeutic drugs were administered weekly or every 3 weeks in the KEYNOTE-040 trial, the cycle length of our model was 3 weeks. Therefore, all costs we provided were for every 3 weeks. All aspects of direct medical costs for treating the disease, including the cost for pembrolizumab or standard therapy, imaging and laboratory tests, hospitalisation, administration for stable state, and the cost for subsequent therapy in progressive disease, were taken into account. Since patients randomised to the SOC arm received one of three chemotherapy regimens, the drug acquisition cost was calculated as a weighted average 


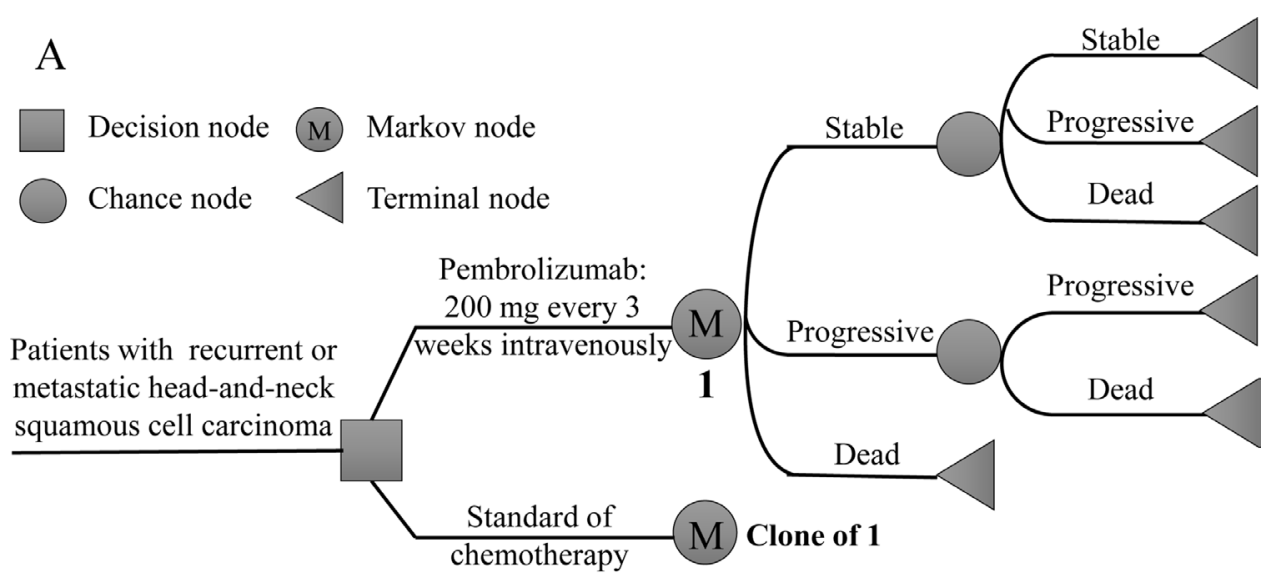

B

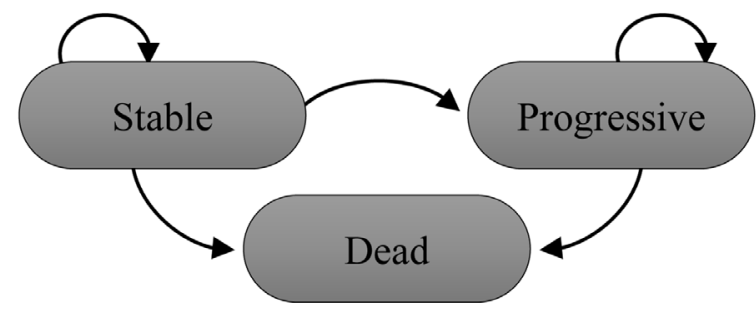

Figure 1 Model structure. (A) Abbreviated decision tree and Markov model; and (B) model states and transitions.

cost based on the patient's number of each regimen in KEYNOTE-040. In addition, time cost was estimated at US $\$ 35.73$ per day on the basis of the average monthly salary in China in 2018. ${ }^{18}$ Supportive care cost and terminal cancer cost were also included and extracted from published articles. ${ }^{19} 20$ The incidence of adverse events comes from the KEYNOTE-040 clinical trial. Costs due to severe (grade 3-4) treatment-related adverse events were either derived from the literature ${ }^{21} 22$ or calculated from the payer's perspective in China. Costs for grade 1-2 adverse events were deemed to be negligible. All costs in the model were adjusted to US dollars based on the 2018 average exchange rate (US\$1=¥6.6174) ${ }^{23}$ and discounted at a rate of $3 \%$ annually (table 1 ).

\section{Utilities and outcome measures}

Effectiveness was measured in quality-adjusted life years (QALY), which is equal to the survival time of the patient in a certain health state multiplied by the health utility value (quality of life weight) during that period. In this study, health utility scores were obtained from the previously published literature, ${ }^{24}$ with an estimation of 0.65 in the stable state per year, 0.52 in the progression state per year and 0 in the death state (table 1 ).

The cost-effectiveness of pembrolizumab versus methotrexate, docetaxel or cetuximab was assessed by incremental cost-effectiveness ratio (ICER), which is expressed as the incremental cost between the two treatment approaches per QALY gained. Treatments were considered 'cost-effective' if the ICER was less than a willingnessto-pay threshold (WTP) of US\$28 130 per QALY. The threshold of US $\$ 28130$ per QALY was three times China's per capita gross domestic product according to the WHO recommendations for cost-effectiveness analysis.

\section{Sensitivity analysis}

Univariate analysis was performed for model parameters subject to uncertainty. The value of parameters was varied one at a time by $\pm 20 \%$ except for discount rate ranging from $0 \%$ to $8 \%$. A tornado analysis was used to rank-order the following parameters in order of potential impact on the outputs. The parameters included cost of pembrolizumab, cost of standard care, cost for stable state, cost for progressive state, probability from stable to progression, probability from stable to death, and utility for stable and progressive state.

In order to evaluate the robustness of the model to further address the uncertainty in model input parameters, probabilistic sensitivity analysis (PSA) was performed using a second-order Monte Carlo simulation. Every time the model was run, all parameters were varied over their defined distribution (gamma distributions for costs and beta distributions for values with a range between 0 and 1 ) simultaneously. The simulation included 1000 iterations.

\section{RESULTS}

\section{Base case}

All patients were dead in both arms at the termination of model simulation. Patients in the pembrolizumab group yielded 0.31 QALYs compared with 0.25 QALYs for patients in the SOC group. The total cost incurred was US\$45 861 in the pembrolizumab group and US\$41 950 in the SOC group. These results led to an ICER of US\$65 186 per QALY higher than WTP, indicating that pembrolizumab is not cost-effective compared with SOC therapy. 
Table 1 Parameters for cost-effectiveness model

\begin{tabular}{|c|c|c|c|c|c|}
\hline \multirow[b]{2}{*}{ Variable } & \multirow[b]{2}{*}{ Value } & \multicolumn{2}{|l|}{ Range } & \multirow[b]{2}{*}{ Distribution } & \multirow[b]{2}{*}{ Source } \\
\hline & & Minimum & Maximum & & \\
\hline \multicolumn{6}{|l|}{ Transition probabilities } \\
\hline \multicolumn{6}{|l|}{ Pembrolizumab } \\
\hline Progression from stable state & 0.281 & 0.225 & 0.337 & Beta & 14 \\
\hline Death from stable state & 0.079 & 0.063 & 0.095 & Beta & 14 \\
\hline Mortality after progression & 0.104 & 0.083 & 0.125 & Beta & 14 \\
\hline \multicolumn{6}{|l|}{ Standard chemotherapy } \\
\hline Progression from stable state & 0.260 & 0.208 & 0.312 & Beta & 14 \\
\hline Death from stable state & 0.096 & 0.076 & 0.115 & Beta & 14 \\
\hline Mortality after progression & 0.140 & 0.112 & 0.168 & Beta & 14 \\
\hline \multicolumn{6}{|l|}{ Utilities } \\
\hline Stable state & 0.650 & 0.500 & 1.000 & Beta & 26 \\
\hline Progressive state & 0.520 & 0.200 & 0.700 & Beta & 26 \\
\hline \multicolumn{6}{|l|}{ Cost } \\
\hline Pembrolizumab & 5421 & 4337 & 6506 & Gamma & Calculated \\
\hline Standard first & 1439 & 1151 & 1727 & Gamma & Calculated \\
\hline Standard & 1253 & 1002 & 1503 & Gamma & Calculated \\
\hline Administration & 13 & 10 & 15 & Gamma & Calculated \\
\hline Test & 154 & 123 & 185 & Gamma & Calculated \\
\hline Time cost & 750 & 600 & 900 & Gamma & Calculated \\
\hline \multicolumn{6}{|l|}{ Severe adverse events } \\
\hline Pembrolizumab & 1227 & 981 & 1472 & Gamma & Calculated \\
\hline Standard & 5855 & 4684 & 7027 & Gamma & Calculated \\
\hline Cancer progression & 2555 & 1677 & 3620 & Gamma & 21 \\
\hline Best supportive care & 157.6 & 126.1 & 191.5 & Gamma & 20 \\
\hline Terminal cancer care & 2039.4 & 1631.5 & 2447.3 & Gamma & 19 \\
\hline
\end{tabular}

\section{Sensitivity analyses}

The results of univariate sensitivity analyses are depicted in the tornado diagram in figure 2. The variables with the most impact on the ICER included utility values for progressive state, probability from stable to progressive in the SOC group, as well as cost of pembrolizumab. Within the $\pm 20 \%$ range of each variable, ICER remained $>$ US $\$ 28$ 130 per QALY.

Finally, PSA over 1000 iterations was performed to vary distributions of cost, survival and utility simultaneously. The cost-effectiveness acceptability curve is displayed in figure 3A. It was demonstrated that standard therapy was more likely to be cost-effective compared with pembrolizumab at a WTP value of US\$28 130 per QALY. The scatterplot of the results of each iteration is shown in figure 3B. The majority of the points were above the WTP threshold line and falling in the first quadrant, indicating that pembrolizumab was not cost-effective versus standard care.

\section{DISCUSSION}

To our knowledge, this is the first cost-effectiveness analysis comparing pembrolizumab with methotrexate, docetaxel or cetuximab in treating patients with $\mathrm{r} / \mathrm{mHNSCC}$. Clinical data were derived from the KEYNOTE-040 trial, which demonstrated improved overall survival for pembrolizumab versus SOC therapy. Model results suggested that if we considered the conventional WTP threshold of US $\$ 28$ 130 per QALY as our cut-off, pembrolizumab was not costeffective compared with standard therapy in $\mathrm{r} / \mathrm{mHNSCC}$, providing an ICER of US\$65 186 per QALY. A large incremental cost and a slight benefit in health outcome led to the high ICER exceeding WTP threshold. Moreover, both univariate sensitivity analysis and PSA demonstrated robust cost-effectiveness results to uncertainty of model input parameters.

Similar economic assessments of pembrolizumab for the treatment of non-small cell lung cancer (NSCLC) in China consistently led to the same conclusion..$^{25}$ An evaluation of pembrolizumab compared with chemotherapy for the treatment of PD-L1-positive NSCLC in China resulted in an ICER of US\$103 128 per QALY. However, the results may be diverse in different countries or cancer types. Georgieva et $a l^{26}$ suggested that first-line pembrolizumab for advanced NSCLC may be cost-effective 


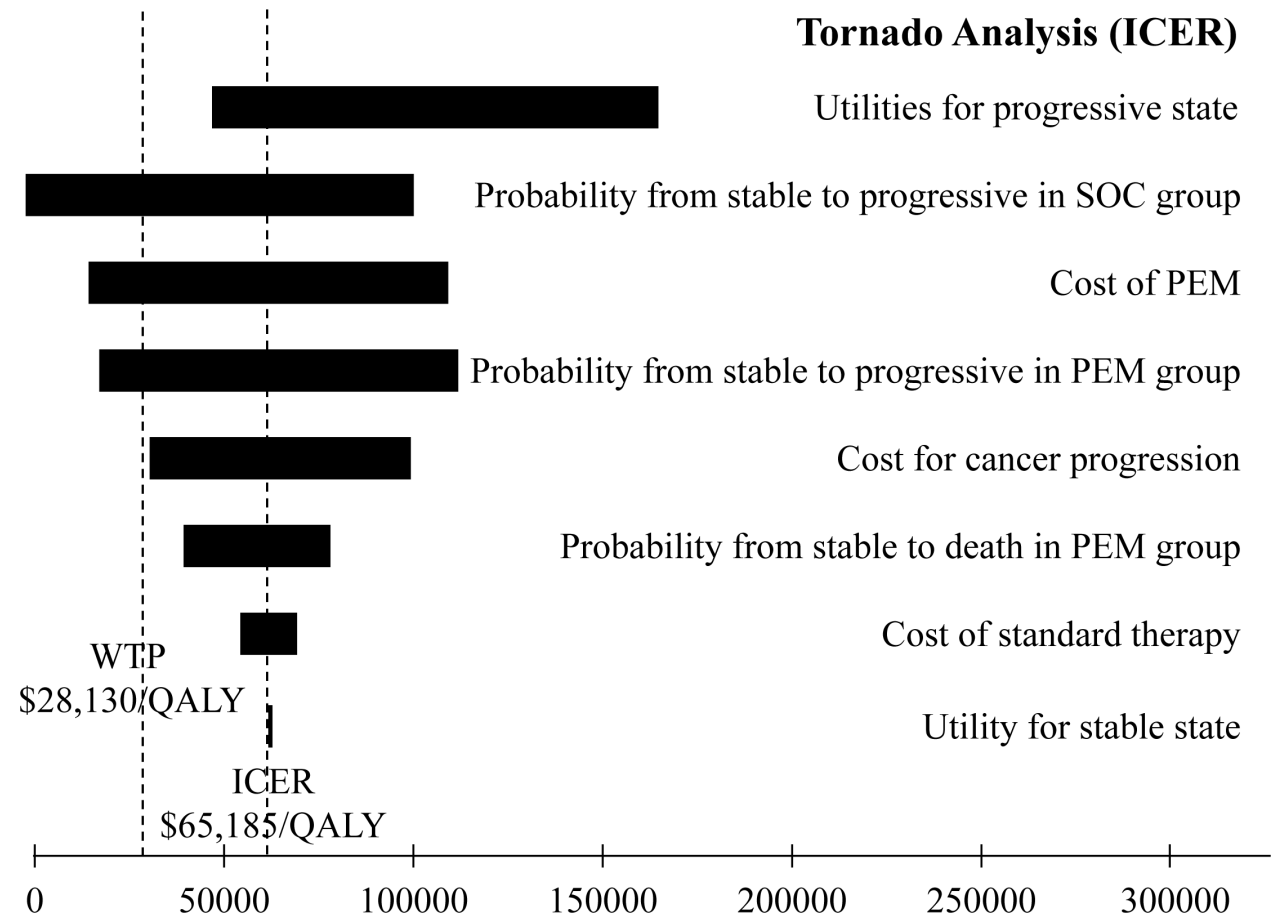

Figure 2 Tornado plot of the univariate sensitivity analyses for pembrolizumab versus standard-of-care therapy. ICER, incremental cost-effectiveness ratio; PEM, pembrolizumab; QALY, quality-adjusted life year; WTP, willingness-to-pay threshold.

compared with platinum-doublet chemotherapy in the USA but not in the UK, despite very similar ICER values in both countries. Sarfaty et $a l^{27}$ found that second-line pembrolizumab for advanced bladder cancer might be considered cost-effective in the USA but not in the UK and Australia. The difference in cost-effectiveness and
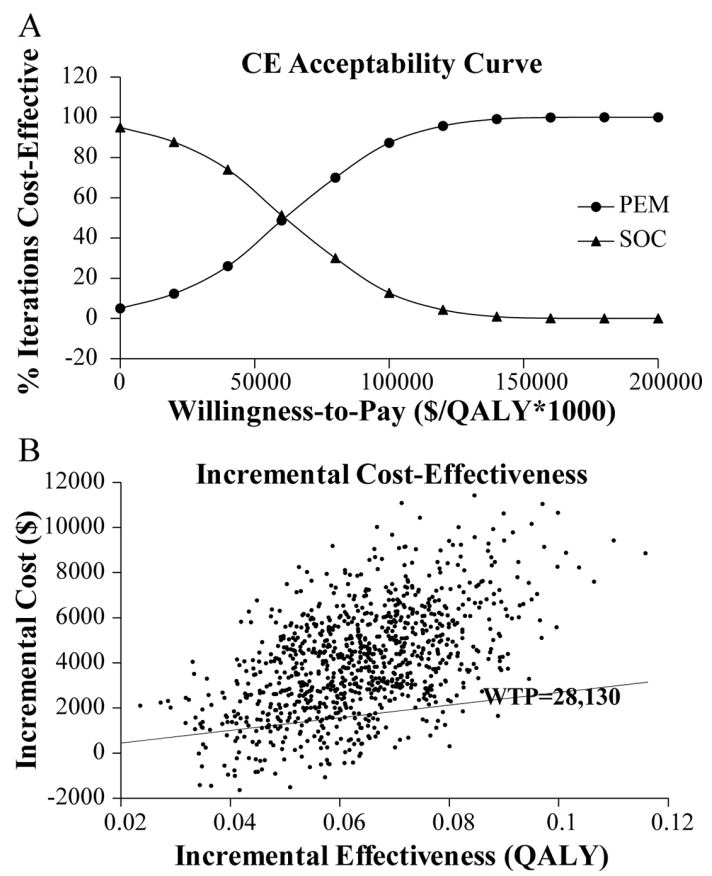

Figure 3 Probabilistic sensitivity analyses for pembrolizumab versus standard-of-care (SOC) therapy. (A) Cost-effectiveness (CE) acceptability curve; and (B) costeffectiveness plane. PEM, pembrolizumab; QALY, qualityadjusted life years; WTP, willingness to pay.
WTP thresholds between countries likely explained the difference in findings related to the cost-effectiveness of pembrolizumab.

This analysis also had several limitations. Like most cost-effectiveness analyses, resources used in clinical trials might not represent resources in actual clinical practice since clinical trials are conducted in a selected population meeting the inclusion and exclusion criteria. Also, since clinical outcomes and utilities were based on previously published studies instead of prospective data, the results in this analysis may be biased. Additionally, pembrolizumab could be a cost-effective strategy for treating patients with $\mathrm{r}$ /mHNSCC in developed countries with WTP thresholds greater than US\$80 000. Finally, although PSA can directly reflect the influence of model uncertainty on the results, it generally assumes that the parameters are independent of each other, which may affect the credibility of the results of pharmacoeconomic evaluation. Considering that the results are still stable when the random simulations are carried out for 1000 times, we believe these are credible.

Despite the above limitations, we still believe that our analysis is reasonable. The analysis was based on the China Guidelines for Pharmacoeconomic Evaluations and Manual ${ }^{28}$ and the joint recommendations of the CostEffectiveness in Health and Medicine. ${ }^{29}$ Furthermore, extensive sensitivity analyses presented in this manuscript were performed to evaluate uncertainty on the outcomes. In conclusion, although pembrolizumab improves overall survival in patients with $\mathrm{r} / \mathrm{mHNSCC}$, this therapy is not a cost-effective strategy compared with standard therapy in China. 
Author affiliations

${ }^{1}$ Department of Pharmacy, The Cancer Hospital of the University of Chinese Academy of Sciences ( Zhejiang Cancer Hospital), Hangzhou, Zhejiang, China ${ }^{2}$ Institute of Basic Medicine and Cancer ( IBMC ) , Chinese Academy of Sciences, Hangzhou, Zhejiang, China

Contributors Study conception and design: WX, HD and GY. Acquisition of data: $Q F, X Z, Y T$ and GX. Analysis and interpretation of data: WX and HD. Drafting of the manuscript: WX and GY. All authors revised the first draft and agreed with the final version.

Funding This research was supported by the Natural Science Foundation of Zhejiang Province (LQ18H160018) and the Natural Science Foundation of China (81903898)

\section{Competing interests None declared.}

Patient consent for publication Not required.

Provenance and peer review Not commissioned; externally peer reviewed. Data availability statement № additional data are available.

Supplemental material This content has been supplied by the author(s). It has not been vetted by BMJ Publishing Group Limited (BMJ) and may not have been peer-reviewed. Any opinions or recommendations discussed are solely those of the author(s) and are not endorsed by BMJ. BMJ disclaims all liability and responsibility arising from any reliance placed on the content. Where the content includes any translated material, BMJ does not warrant the accuracy and reliability of the translations (including but not limited to local regulations, clinical guidelines, terminology, drug names and drug dosages), and is not responsible for any error and/or omissions arising from translation and adaptation or otherwise.

Open access This is an open access article distributed in accordance with the Creative Commons Attribution Non Commercial (CC BY-NC 4.0) license, which permits others to distribute, remix, adapt, build upon this work non-commercially, and license their derivative works on different terms, provided the original work is properly cited, appropriate credit is given, any changes made indicated, and the use is non-commercial. See: http://creativecommons.org/licenses/by-nc/4.0/.

\section{ORCID iDs}

Wenxiu Xin http://orcid.org/0000-0001-5491-4930

Yinghui Tong http://orcid.org/0000-0001-5060-3975

\section{REFERENCES}

1 Ferlay J, Soerjomataram I, Dikshit R, et al. Cancer incidence and mortality worldwide: sources, methods and major patterns in GLOBOCAN 2012. Int J Cancer 2015;136:E359-86.

2 Fitzmaurice C, Allen C, Stanaway J, et al. Global, regional, and National cancer incidence, mortality, years of life lost, years lived with disability, and Disability-Adjusted life-years for 32 cancer groups, 1990 to 2015: a systematic analysis for the global burden of disease study. JAMA Oncol 2017;3:524-48.

3 Beitler JJ, Zhang Q, Fu KK, et al. Final results of local-regional control and late toxicity of RTOG 9003: a randomized trial of altered fractionation radiation for locally advanced head and neck cancer. Int J Radiat Oncol Biol Phys 2014;89:13-20.

4 Ang KK, Zhang Q, Rosenthal DI, et al. Randomized phase III trial of concurrent accelerated radiation plus cisplatin with or without cetuximab for stage III to IV head and neck carcinoma: RTOG 0522. J Clin Oncol 2014;32:2940-50.

5 Vermorken JB, Specenier P. Optimal treatment for recurrent/ metastatic head and neck cancer. Annals of Oncology 2010;21:vii252-61.

6 Stewart JSW, Cohen EEW, Licitra L, et al. Phase III study of gefitinib compared with intravenous methotrexate for recurrent squamous cell carcinoma of the head and neck [corrected]. J Clin Oncol 2009;27:1864-71.

7 Argiris A, Ghebremichael M, Gilbert J, et al. Phase III randomized, placebo-controlled trial of docetaxel with or without gefitinib in recurrent or metastatic head and neck cancer: an eastern cooperative Oncology Group trial. J Clin Oncol 2013;31:1405-14.

8 Soulières D, Faivre S, Mesía R, et al. Buparlisib and paclitaxel in patients with platinum-pretreated recurrent or metastatic squamous cell carcinoma of the head and neck (BERIL-1): a randomised, double-blind, placebo-controlled phase 2 trial. Lancet Oncol 2017;18:323-35.

9 Vermorken JB, Trigo J, Hitt R, et al. Open-Label, uncontrolled, multicenter phase II study to evaluate the efficacy and toxicity of cetuximab as a single agent in patients with recurrent and/or metastatic squamous cell carcinoma of the head and neck who failed to respond to platinum-based therapy. J Clin Oncol 2007;25:2171-7.

10 Motzer RJ, Tannir NM, McDermott DF, et al. Nivolumab plus ipilimumab versus sunitinib in advanced renal-cell carcinoma. $N$ Eng J Med 2018;378:1277-90.

11 Vaughn DJ, Bellmunt J, Fradet $\mathrm{Y}$, et al. Health-Related quality-of-life analysis from KEYNOTE-045: a phase III study of pembrolizumab versus chemotherapy for previously treated advanced urothelial cancer. JCO 2018:36:1579-87.

12 Carlino MS, Long GV, Schadendorf D, et al. Outcomes by line of therapy and programmed death ligand 1 expression in patients with advanced melanoma treated with pembrolizumab or ipilimumab in KEYNOTE-006: a randomised clinical trial. Eur J Cancer 2018;101:236-43.

13 Mok TSK, Wu Y-L, Kudaba I, et al. Pembrolizumab versus chemotherapy for previously untreated, PD-L1-expressing, locally advanced or metastatic non-small-cell lung cancer (KEYNOTE-042): a randomised, open-label, controlled, phase 3 trial. Lancet 2019;393:1819-30.

14 Cohen EEW, Soulières D, Le Tourneau C, et al. Pembrolizumab versus methotrexate, docetaxel, or cetuximab for recurrent or metastatic head-and-neck squamous cell carcinoma (KEYNOTE-040): a randomised, open-label, phase 3 study. Lancet 2019;393:156-67.

15 Ellis MJ, Llombart-Cussac A, Feltl D, et al. Fulvestrant 500 Mg versus anastrozole $1 \mathrm{Mg}$ for the first-line treatment of advanced breast cancer: overall survival analysis from the phase II first study. J Clin Oncol 2015;33:3781-7.

16 Sonnenberg FA, Beck JR. Markov models in medical decision making: a practical guide. Med Decis Making 1993;13:322-38.

17 Chan JK, Herzog TJ, Hu L, et al. Bevacizumab in treatment of HighRisk ovarian Cancer-A Cost-Effectiveness analysis. Oncologist 2014;19:523-7.

18 Huang J, Liao W, Zhou J, et al. Cost-Effectiveness analysis of adjuvant treatment for resected pancreatic cancer in China based on the ESPAC-4 trial. Cancer Manag Res 2018;10:4065-72.

19 Wu B, Yao Y, Zhang K, et al. Ras testing and cetuximab treatment for metastatic colorectal cancer: a cost-effectiveness analysis in a setting with limited health resources. Oncotarget 2017;8:71164-72.

20 Wu B, Zhang Q, Sun J. Cost-Effectiveness of nivolumab plus ipilimumab as first-line therapy in advanced renal-cell carcinoma. $J$ Immunother Cancer 2018;6:124.

21 Wu B, Dong B, Xu Y, et al. Economic evaluation of first-line treatments for metastatic renal cell carcinoma: a cost-effectiveness analysis in a health resource-limited setting. PLoS One 2012;7:e32530

22 Wu B, Ye M, Chen $\mathrm{H}$, et al. Costs of trastuzumab in combination with chemotherapy for HER2-positive advanced gastric or gastroesophageal junction cancer: an economic evaluation in the Chinese context. Clin Ther 2012;34:468-79.

23 Zhang P-F, Xie D, Wen F, et al. Lenalidomide plus rituximab vs rituximab alone in relapsed or refractory indolent lymphoma: a costeffectiveness analysis. Cancer Med 2020;9:5312-9.

24 Hannouf MB, Sehgal C, Cao JQ, et al. Cost-Effectiveness of adding cetuximab to platinum-based chemotherapy for first-line treatment of recurrent or metastatic head and neck cancer. PLoS One 2012;7:e38557

25 Liao W, Huang J, Hutton D, et al. Cost-Effectiveness analysis of firstline pembrolizumab treatment for PD-L1 positive, non-small cell lung cancer in China. J Med Econ 2019;22:344-9.

26 Georgieva M, da Silveira Nogueira Lima JP, Aguiar P, et al. CostEffectiveness of pembrolizumab as first-line therapy for advanced non-small cell lung cancer. Lung Cancer 2018;124:248-54.

27 Sarfaty M, Hall PS, Chan KKW, et al. Cost-Effectiveness of pembrolizumab in second-line advanced bladder cancer. Eur Urol 2018;74:57-62.

28 Liu SH GE, Wu JH, Wu J, et al. China guidelines for pharmacoeconomic evaluations and manual. Beijing: Science Press, 2015.

29 Shepard DGold MR, Russell LB, Weinstein MC, eds. CostEffectiveness in health and medicine. New York: Oxford University Press, 1996. 